\title{
Water Activity in Biological Systems: a Dew-point Method for Its Determination
}

\author{
By G. D. ANAGNOSTOPOULOS \\ Microbiology Department, Queen Elizabeth College, University of London, \\ Campden Hill, London, W. 8
}

(Received 13 February 1973)

In hydrated systems it is the state of the water, expressed as water activity $\left(A_{\mathrm{w}}\right)$, rather than the absolute water content per se that determines more precisely its biological status.

A common principle of the methods used to determine $A_{\mathrm{w}}$ is that a sample of material is allowed to reach equilibrium with either (a) its overhead atmosphere in a confined space (e.g. Mossel \& Kuikj, I955; Ayerst, I965; Bogsanyi \& Weeden, 1968) whose equilibrium relative humidity is then measured, or (b) a stream of gas of known relative humidity followed by analytical determination of the moisture content of the sample (e.g. Loncin, Bimbenet \& Lenges, I968). The present method belongs to category (a) whereby the water vapour pressure exerted by a system is determined and expressed as a proportion of the vapour pressure exerted by pure water at the same temperature.

\section{Description of apparatus}

The method is based on dew-point determination and the apparatus shown in Fig. $I$ is basically an equilibration vessel housing the dew-point module and the thermocouple probes. Other parts of the system include a water bath, a remote reading electronic thermometer, a magnetic stirrer and a microscope lamp.

The equilibration vessel is a $100 \mathrm{ml}$ cylindrical chemostat vessel (Pirt, \& Callow, 1964) 5.5 by $9.0 \mathrm{~cm}$ with a water jacket over $7.5 \mathrm{~cm}$ of its length leaving a $\mathrm{I} .5 \mathrm{~cm}$ neck. Suspended from the rubber stopper are: $(a)$ a magnetic air circulation fan, $(b)$ a 'frigistor' module, and (c) two thermocouple probes, one for the air temperature and the other for the dew point.

The water circulation system is flexibly extended from the top outlet of the vessel jacket to its counterpart on the rubber stopper and through the stopper into the equilibration vessel where it forms a brick-shaped radiator. This heat radiator also plays the role of the "heat sink' that is required by the frigistor.

Attached to a small ball bearing $(6 \mathrm{~mm}$ diam. $)$ is the air circulation fan made of a thin aluminium disk cut and twisted radially with the leading edge of the resulting blades pointing up; its rotation is effected by a bar magnet clipped on its lower surface. The whole assembly is suspended on a stainless steel rod firmly inserted into the centre of the rubber stopper.

The 'frigistor' (Mectron (Frigistor) Ltd, Slough, Buckinghamshire) which, as indicated by Norrish (1969), can be used preferentially in confined spaces on account of its small size $(2 \times 0.9 \times 0.5 \mathrm{~cm})$ is a thermoelectric cooling module utilizing the Peltier effect. When a d.c. current ( 2 to 4 A) flows through the module, heat is transferred from one surface of the block to the opposite surface, i.e. one face cools as the other heats up. The excess of heat generated on one surface is dissipated by contact with the 'heat sink', i.e. the radiator of the water circulation system, upon which the module is firmly cemented with araldite. A thin $(0.5 \mathrm{~mm})$ stainless steel mirror, insulated over $2 / 3$ of its outermost surface and leaving a $7 \times 9 \mathrm{~mm}$ dew 

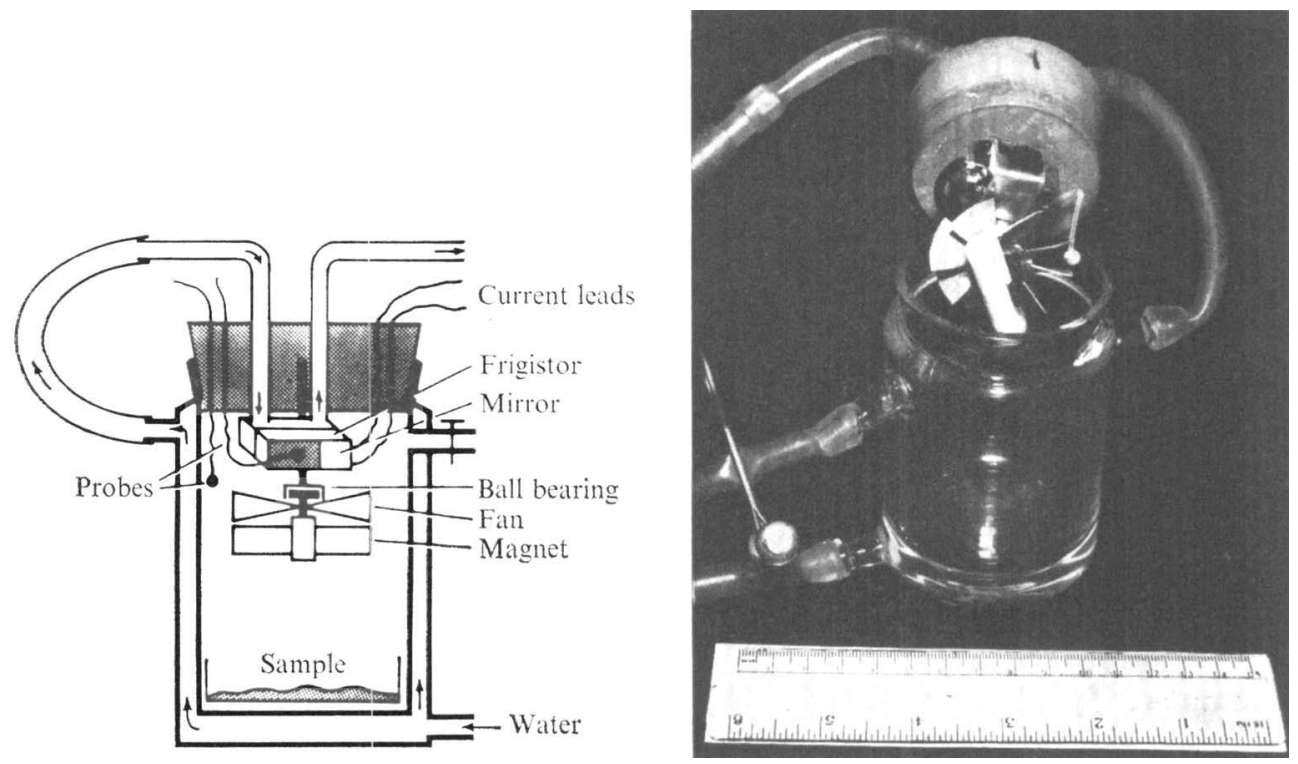

Fig I. Illustration of the apparatus and its handling without discontinuing the water circulation.

area, is similarly cemented on the cool surface of the frigistor. The exposed area of the mirror is peripherally lacquered to provide contrast to the actual dew area which is confined in a central square about $5 \times 5 \mathrm{~mm}$. Of the two 'small surface' temperature probes (no. 427 Yellow Spring Instrument Co.), one is solder padded on the mirror surface concealed and held in position by the insulation cover and the other is freely suspended from the rubber stopper.

\section{Operation}

A $5 \mathrm{~cm}$ plate carrying the sample of material (about $2 \mathrm{~g}$ ) is inserted into the vessel horizontally, pressed with a finger against the inside wall and slid down gently. The rubber stopper is then replaced and the excess pressure released from the side overflow of the original fermentor. The fan is then switched on and the system is allowed $15 \mathrm{~min}$ to equilibrate. For the dew point determination the frigistor is switched on, the mirror is illuminated with a microscope lamp and, when the dew appears on the mirror, its temperature is read on the instument. Normally the air temperature before and after a reading is identical owing to the efficient air circulation and the temperature control system. Condensation on the surfaces of the apparatus, which is an important drawback in dew-point methods, does not occur in this apparatus and the degree of condensation on the mirror surface is effectively controlled.

An important advantage of the apparatus is that by using a suitable switch (e.g. a remote push button switch) the operator is able to control the current flow through the frigistor, e.g. in small amounts to slow down the cooling rate of the mirror and bring the temperature pointer of the instrument to a standstill when the reading is about to be taken (when dew appears on the mirror surface). This means high precision of reading and avoids excessive condensation, two requirements not easily met by other methods.

As the accurate determination of the dew point depends on the detection of the first trace of dew formation, the apparatus has obvious advantages especially over methods using the 
Renault tube principle. Precise control of the cooling rate and ability to stabilize the temperature when necessary enable even inexperienced operators to determine accurately the temperature at which the first appearance or disappearance of dew occurs to within about $0 \cdot{ }^{\circ} \mathrm{C}$. With any given sample, these two phenomena occur within a critical range of $0 \cdot \mathrm{I}^{\circ} \mathrm{C}$, providing an objective criterion for measurements. The circulating water, if free from air bubbles, and the double wall of the vessel provide a viewer through which a crystal-clear view of the illuminated mirror is obtained.

Each measurement takes only a very short time so that several measurements on the same sample can be made within a few minutes. The data are processed as usual. The maximum water pressure vapour at the two temperatures, i.e. air temperature $\left(p_{0}\right)$ and dew point $(p)$ are obtained from standard tables and the $A_{\mathrm{w}}$ of the medium derived from the equation $A_{\mathrm{w}}=p / p_{0}$. The accuracy of the results depends mainly on the accuracy and readability of the temperature instrument. The instrument used with this apparatus (Y.S.I. Model 46) has a readibility $0.05{ }^{\circ} \mathrm{C}$ equivalent to $0.0025 A_{\text {w }}$ which compares favourably with previously published methods. This is mainly due to the fine control of the cooling rate of the mirror afforded by the operational principle of the cooling module.

Successive readings are close to each other (usually within \pm 0.003 ). With solutions containing different concentrations of glycerol, $A_{\mathrm{w}}$ values within the range 0.750 to $0.990 \mathrm{ob}-$ tained with the present apparatus were within \pm 0.003 of those quoted by Scott (1957). These differences may be due to slight differences in molalities of our solutions and/or to actual differences in $A_{\text {w }}$ measurements although $A_{\text {w }}$ values of stock solutions determined on different days were found to be almost identical as far as the third and sometimes the fourth decimal points.

\section{REFERENCES}

AYERST, G. (1965). Determination of the water activity of some hygroscopic food materials by a dew-point method. Journal of the Science of Food and Agriculture 2, 7 $71-78$.

BogsanYi, D. J. \& WeEDEN, D. G. (I968). A simple apparatus for water vapour pressure-moisture studies by a dew point method. Chemistry and Industry, 741-742.

LONCIN, M., BIMBENET, J. J. \& LenGes, J. (I968). Influence of the activity of water on the spoilage of foodstuffs. Journal of Food Technology 3, 131-I 42.

Mossel, D. A. A. \& van KuiJK, H. J. L. (I955). A new and simple technique for the direct determination of the equilibrium relative humidity of foods. Food Research 20, 415-423.

NORRISH, R.S. (1969). A study of the rate of gain or loss of moisture from sugar and sugar confectionery products during storage. Research Report No. I44, 23-25. Leatherhead, Surrey: The British Food Manufacturing Industries Research Association.

PIRT, S. J. \& CALlow, D. S. (1964). Continuous flow culture of the ERK and L types of mammalian cells. Experimental Cell Research 33, 413-421.

Scort, W. J. (1957). Water relations of food spoilage micro-organisms. Advances in Food Research 7, 83-127. 